\title{
Palliative Sedation of Terminally ill Patients
}

\author{
Fabíola Leite Nogueira ${ }^{1}$, Rioko Kimiko Sakata, TSA ${ }^{2}$
}

Summary: Nogueira FL, Sakata RK - Palliative Sedation of Terminally ill Patients.

Background and objectives: Although there is extensive discussion on the subject in medical literature, most of the questions still unanswered due to the lack of clear definitions and guidelines, in addition to a large number of contradictions in literature. The aim of this review is to try to determine the best form of sedation for patients with cancer.

Content: The following data on sedation were collected: definitions, classifications, major events and indication criteria, selection of drugs used, drugs most used, doses and routes, duration of sedation, nutritional support and hydration during sedation, and sedation at home.

Conclusions: Although in recent years palliative sedation is being considered a normal medical practice, there are still many gaps in our current understanding. There is no consensus about which are the standard drugs, maintenance or not of food, fluid intake, and hydration. Moreover, there is no ethical clarification on possible life-shortening effects and decision-making process.

Keywords: Deep Sedation; Neoplasms; Palliative Care.

@2012 Elsevier Editora Ltda. All rights reserved.

\section{INTRODUCTION}

The terminally ill patient is one whose disease is irreversible. Palliative treatment is indicated for patients with end stage advanced disease, and when there is no possibility of restoring the patient's health and imminent death seems inevitable and predictable. However, there are doubts about the timing, medication, dose, and route to be used for palliative sedation.

Sedative medications may be used to reduce patient's awareness, provide comfort and relieve his unbearable anxiety 1,2 . Sedation of terminally ill patient has been debated for many years, both in the field of palliative care and elsewhere. Some authors describe terminally ill patient's sedation as a form of slow euthanasia or mercy ${ }^{3,4}$. According to these authors, sedation is associated with significant ethical issues. Despite extensive discussion on the subject in medical literature, most questions still unanswered due to lack of clear definitions and guidelines, in addition to the large number of contradictions in literature 4,5 .

According to the European Association of Palliative Care (EAPC), "palliative sedation" of terminally ill patients should be distinguished from euthanasia. In palliative sedation, the goal

\footnotetext{
Received from the Department of Anesthesiology, Pain and Intensive Care - Universidade Federal de São Paulo, Brazil.

1. ME-4 in Pain; Specialization in Anesthesiology.

2. PhD, Coordinator, Department of Pain.

Submitted on February 8, 2011.

Accepted on September 5, 2011.

Correspondence to:

Dra. Rioko Kimiko Sakata

Rua Três de Maio 61/apto51

Vila Clementino

04044020 - São Paulo, SP, Brazil

E-mail: riokoks.dcir@epm.br
}

is to alleviate suffering using only sedatives titrated to control symptoms. In euthanasia, the intention is to take the life of the patient by administering a lethal drug ${ }^{1,6}$. It means that palliative sedation, correctly indicated and in a proper dose is not a "shortcut" to achieve the same goal of euthanasia ${ }^{3}$. There is no evidence that palliative sedation appropriately administered shorten the life. For palliative sedation, it is very important that consciousness be reduced only to a level sufficient to relieve symptoms, which is individual and can vary widely ${ }^{3}$.

\section{Definitions}

Many terms are used in studies of sedation in patients under palliative care, and they are often confusing. Many terms are used interchangeably, complicating the research literature ${ }^{4,7}$. The terms used to indicate the use of palliative sedation therapy often cause doubt, sometimes it is for "unbearable symptoms" and others for "refractory symptoms".

Some authors have criticized the use of "terminal sedation" as a synonym for "palliative sedation", considering that the former can be misinterpreted as if sedation means "terminate" the life and not "relieve" the symptoms, as suggested by the latter ${ }^{3,7}$.

Other authors have proposed specific definitions for palliative sedation and the symptoms indicating its use. Palliative sedation, therefore, would be the use of sedative drugs to relieve the symptoms that are unbearable and refractory to other treatments, by reducing the level of consciousness. Unbearable symptoms should be determined based on patient outcomes, in conjunction with medical staff, caregivers, and family members. Symptoms would be defined as refractory when all other forms of treatment have failed, without compromising the patient's level of consciousness ${ }^{4,7}$. 
Palliative sedation therapy is defined as the use of specific sedative drugs to relieve the unbearable suffering caused by refractory symptoms, by reducing the patient's consciousness, using careful titration of drugs ${ }^{8}$.

Palliative sedation is not euthanasia because it is an intervention performed with the intent to provide relief of symptoms, and the patient's death is not a success criterion ${ }^{8}$.

Palliative sedation can be classified according to the degree "mild or conscious" when consciousness is maintained, allowing communication of the patient, or "deep" when the patient is unconscious or semiconscious. According to the duration, it is classified as "intermittent" when the patient has some periods of consciousness, or "continuous" when the patient is unconscious until death 7 . To indicate continuous deep sedation, the disease should be irreversible and advanced, with death expected within hours or days ${ }^{8}$. Palliative sedation does not shorten survival time ${ }^{9}$.

\section{Survival after the onset of sedation}

Some authors compared the survival time between sedated and non-sedated patients, finding no statistical difference in the outcomes ${ }^{4,10,11}$. In a retrospective study, however, survival was significantly higher in patients who received sedation during the last week of life than in those sedated only in the last 48 hours of life ${ }^{11}$. Furthermore, another study found no correlation between the survival time of sedated patients and drugs used ${ }^{4}$. In a palliative care unit, survival was higher in sedated than in non-sedated patients ${ }^{12}$.

\section{Indications}

The main indications for sedation are pain, delirium, agitation, and dyspnea ${ }^{3,12-16}$. Usually, patients present with at least two refractory symptoms, and the most important are pain, fatigue, vomiting, depression, drowsiness, tiredness, seizures, bleeding, insomnia, suffering, distress, and malaise 9,15,17-19. Sedation is indicated in the progression of cancer with cachexia and organ failure and when palliative treatments such as steroids for fatigue, opioids for dyspnea, antisecretory to bronchial secretion, antipsychotic for delirium, and opioids for pain have failed ${ }^{17}$.

There are some criteria to indicate the patient's terminal sedation, which must be strictly followed in order to alleviate only the patient's suffering (not family) and not anticipate his death ${ }^{4,20}$.

A survey was conducted with neurologists of the American Academy to learn their opinion regarding sedation. Among the physicians who responded, $96 \%$ believe that the goal of sedation is to relieve suffering, $85 \%$ do not consider it equivalent to euthanasia, and $92 \%$ considered it acceptable for patients with terminal cancer ${ }^{21}$.

The indication must be defined, preferably by the multidisciplinary team, if the disease is irreversible and death is expected within one-two weeks ${ }^{3}$. The decision to start treatment with sedative drugs must comply with the wishes of the patient, family, or legal guardian and in consensus with medical team. Contraindications, goals, and expectations regarding sedation should be discussed with the family, care team, and patient, if possible, and the possibility of suspension or intermittent sedation should be clarified. Everyone involved in patient care should be informed and aggregated. Family members should be encouraged to stay with the patient. Psychological support should be offered to patients and families with indication of palliative sedation. The support during the grieving process should be continued after death. The care team continues to evaluate the risks and needs, considering the spiritual, emotional, cultural, and social aspects in compliance with the conditions and requests of the family 20 .

All stages of the care process should be clearly described in patient's chart, and it is recommended that documented consent be obtained from the patient or legal guardian ${ }^{20}$.

The events for which relief is sought must be unbearable to the patient, and should also be clearly defined and understood as part of the disease by health professionals. In one study, based on palliative prognostic index, the survival rate of $94 \%$ of patients would be less than three months ${ }^{17}$.

Palliative sedation is only administered when refractory pain is evident and to patients with no prospects ${ }^{9}$. The medical staff following the patient must have enough experience to know when the symptom is refractory ${ }^{8}$.

\section{Decision-making}

Sedation should be considered and discussed between health professionals, patient and his family, faced with a situation of serious suffering caused by refractory symptoms. The patient's condition should be thoroughly evaluated. The decision of administering or not sedation involves the level of sedation (superficial or deep), drug of choice, and dosage ${ }^{3}$.

Regarding freedom and the right to autonomy, the authors recommend an informed consent form addressed to the responsible physician, signed by an adult, in accordance with legal requirements, in which he declares to be aware of the indications, risks, and care given, allowing the palliative treatment with sedatives. Consent form must also contain a request specifying the measures that should not to applied such as cardiopulmonary resuscitation, dialysis, connection to a ventilator, and blood transfusion ${ }^{20}$.

In some countries, such as the Netherlands, the law allows, in cases of disagreement between medical staff and family, that the physician has the final say in making that decision ${ }^{3}$. In life-threatening situations, such as respiratory failure due to tumor growth or bleeding, the medical staff may decide to initiate palliative sedation without family consultation ${ }^{3}$.

Specific criteria for palliative sedation are not fully defined in literature, but all of the following must be respected:

- Presence of a terminal illness with at least one refractory symptom;

- Running out of treatment options for the symptom, including depression, delusions, and anxiety treatments; 
- Assessment of spiritual matters by a clinical specialist or clergy member;

- Debate on continuity of nutritional support and hydration;

- Obtaining informed consent;

- Written order for no resuscitation 22.

\section{Selection of drugs}

The beginning of palliative sedation is an emotionally charged occasion for the family, especially if it rapidly induces loss of consciousness and communication. Medical interventions are sometimes need at the beginning of sedation. In subsequent phases, the administration of drugs can be handed to a nurse or caregiver ${ }^{3}$. Once sedation has been determined, the following principles should be followed ${ }^{22}$ :

- Choose the appropriate agent to start sedation, considering the symptoms presented by the patient;

- Monitor the level of sedation, using specific scales, such as Ramsay;

- Titrate sedative dose to obtain the desired level of sedation;

- Give additional bolus doses or associate other drugs to maintain the achieved level.

The choice of drug to be used for sedation in most cases depends on the professionals' experience and institution. The most used drugs are benzodiazepines, especially midazolam, and barbiturates. Other drugs widely used are neuroleptics 22. Benzodiazepines have been used alone or in combination with neuroleptics and opioids ${ }^{13}$.

In a review of sedation at home, $93 \%$ of patients received midazolam and $7 \%$ levomepromazine ${ }^{14}$. In another review, the drugs were midazolam, morphine, haloperidol, promethazine, and transdermal fentanyl ${ }^{15}$. In a palliative care unit, $70 \%$ of patients received benzodiazepines, but only $3.1 \%$ received continuous deep sedation ${ }^{23}$.

\section{Benzodiazepines}

Benzodiazepines are considered first-choice drugs in the absence of delirium. They should be administered subcutaneously or intravenously by intermittent bolus or continuous infusion ${ }^{24}$.

Midazolam is the most used drug for palliative sedation and is considered a first-choice drug 8,18,25. We found no study comparing midazolam with other drugs. Due to its favorable pharmacodynamic characteristics, it is supposed to have advantages over other benzodiazepines. Midazolam is a greatanxiolytic, has anticonvulsant effect, promotes amnesia, and causes few adverse effects. Its pharmacokinetic characteristics are also favorable. Its half-life is short (an important factor, especially in intermittent sedation), dosage is easily titrated, can be combined with other drugs used in palliative care, and yet can be administered parenterally. Moreover, its effect can be antagonized by flumazenil ${ }^{4,24}$. In a palliative care unit, midazolam was used in $10 \%$ of patients for sedation ${ }^{16}$.

Benzodiazepines were administered orally or sublingually in $71.8 \%$ of patients ${ }^{23}$. Lorazepam was administered mainly for anxiety and agitation, oxazepam to induce sleep, and midazolam for sedation ${ }^{23}$. Agitation caused by delirium can be relieved with midazolam ${ }^{12}$. Diazepam and clonazepam are prescribed for prevention or treatment of seizures ${ }^{23}$.

The use of benzodiazepines for sedation, however, does not always imply adequate control of symptoms ${ }^{26}$.

\section{Opioids and tramadol}

Patient's symptoms must be taken into account when choosing sedatives. If the patient is experiencing pain, which is the most common symptom in end-stage patients, the use of opioids is indicated for relief. Sometimes, opioids are used alone for sedation ${ }^{13}$. When the patient is experiencing pain, this drug can be sufficient to relieve pain and promote palliative sedation. The action of this class of drug is synergic with most hypnotics and neuroleptics. The most appropriate choice of opioid should be based, in principle, on pain intensity. Among the available opioids, morphine stands out as the gold standard for treating moderate and severe pain, according to literature ${ }^{25}$. In a palliative care unit, patients received intravenous morphine more frequently ${ }^{27}$.

Some opioids should not be used. Propoxyphene, a synthetic derivative of methadone, has weak opioid action and long half-life. It is metabolized in the liver to yield norpropoxyphene, which can cause increased excitability of central nervous system, causing tremors, myoclonus, and seizures. Meperidine is an opioid with erratic oral absorption, has a relatively short half-life and toxic metabolites. This drug is metabolised in the liver to yield normeperidine, which has half the analgesic potency of the parent compound, but causes twice as much toxic effects on central nervous system. The neurotoxicity of normeperidine can occur rapidly, especially in patients with renal insufficiency. Other factors that may contribute to central nervous system excitability induced by meperidine are prolonged treatment, high doses, history of seizures, and concomitant administration of other potentially neurotoxic agents ${ }^{28}$.

The use of tramadol, a week opioid agonist and serotonin and norepinephrine reuptake inhibitor, as a palliative drug is limited by its power (more than $600 \mathrm{mg}^{\text {dday }}{ }^{-1}$ are not recommended). However, it may be used for non-severe pain in combination with other drugs.

Transdermal fentanyl is not indicated for sedation, but there is report of its use in literature ${ }^{25}$. When the patient is receiving analgesia with this drug, it should be replaced by intravenous morphine. 
Opioid administration in order to produce only sedation in a patient experiencing no pain is considered inappropriate because sedation may occur associated with undesirable effects, or even not be achieved despite the use of large doses ${ }^{3,29}$. When the patient is experiencing pain, which is present in most patients with terminal cancer, morphine is the first drug to be administered and, sometimes, sufficient for analgesia and sedation. The administration of opioid did not change the duration of sedation ${ }^{12}$.

\section{Other}

Sedation of delirious patients should be considered only after treatment with neuroleptics. In refractory cases, the association of benzodiazepines and neuroleptics should be considered. The neuroleptics used are chlorpromazine ${ }^{18}$, levomepromazine ${ }^{24}$, and haloperidol $18,25,30$. Other drugs used are promethazine ${ }^{25}$ and scopolamine ${ }^{18}$. For patients with intense agitation, barbiturates or propofol may be used ${ }^{24}$.

If adequate sedation is not achieved, other drugs may be associated in order to maximize the effect ${ }^{22,24}$. Drug combination is very useful, but requires reduction of doses used due to the synergistic effect of most sedatives. Antidopaminergic and antihistamines, sedative drugs that also have antiemetic effect, are often used ${ }^{26}$. There is report of a number of cases using ketamine in combination with opioids and benzodiazepines for sedation in terminally ill patients ${ }^{31,32}$ and a case report of its use as monotherapy for palliative sedation ${ }^{33}$.

The symptoms are sometimes refractory to benzodiazepines and antipsychotics, which requires alternative medication. Propofol, a sedative and hypnotic drug, can provide relief from agitation and refractory fatigue ${ }^{34}$. In a systematic review, only four articles were found reporting the favorable effect of propofol for sedation in the last days of life, but all of them are only case reports ${ }^{35}$. This drug was administered when other drugs have failed.

\section{Dose and route of administration}

Drugs may be administered by intravenous, subcutaneous, oral (until the patient loses consciousness), enteral, sublingual, and rectal routs, depending on the route that has been used ${ }^{22}$.

The initial dose of sedatives may be small, with the patient able to communicate regularly ${ }^{8}$. Dose of medication should be increased gradually as needed ${ }^{36}$.

Literature is limited and there is no consensus on the dose of sedatives, but some authors recommend the following drugs to induce and maintain sedation 14,17,22,27:

- Morphine: 20-80 mg.day ${ }^{-1}$.
- Midazolam: 0.5-0.7 mg. $\mathrm{kg}^{-1}$, followed by infusion of 0.5-2 mg.h $\mathrm{h}^{-1} \mathrm{IV}$ or $10 \mathrm{mg}$ followed by $1-6 \mathrm{mg} \cdot \mathrm{h}^{-1}$ subcutaneously.

- Chlorpromazine: $10-25 \mathrm{mg} .(2-8) \mathrm{h}^{-1}$ by oral, sublingual, or rectal routs, or intravenous infusion.

- Haloperidol: 0.5-5 mg.(2-4). $\mathrm{h}^{-1}$ orally or subcutaneously, or infusion of 1-5 mg.day ${ }^{-1}$.

- Pentobarbital: $2-3 \mathrm{mg} \cdot \mathrm{kg}^{-1}$ intravenously, followed by infusion of $1 \mathrm{mg} \cdot \mathrm{kg}^{-1}$.

- Phenobarbital: $200 \mathrm{mg}$ intravenous or subcutaneous bolus injection followed by $600-1,600$ mg.day $^{-1}$.

- Thiopental: 5-7 mg. $\mathrm{kg}^{-1}$ intravenous bolus injection followed by infusion of $70-180 \mathrm{mg} \cdot \mathrm{h}^{-1}$.

- Levomepromazine: $12,5-25$ mg.d-1 subcutaneously.

Regarding dose escalation of sedative medications, there are no protocols or guidelines; however, dose should not be increased unless there is evidence of inadequate sedation ${ }^{22}$. Higher doses of midazolam were necessary for sedation of young people who had used the drug previously and for log time ${ }^{37}$. The initial dose of opioids depends on patients' history of analgesics use ${ }^{38}$.

The routes of administration were subcutaneous and intravenous infusions ${ }^{17}$. The most common route of administration is intravenous, as it quickly provides adequate plasma levels that can be maintained.

\section{Duration of Sedation}

The duration of sedation is variable. In a review of sedation at home, the authors report that it ranges from one to more than one week ${ }^{9,12-15,25,36}$.

The time for symptom management was 24 hours ${ }^{15}$.

Sedation does not necessarily mean that the patient will be maintained sedated until death. In a palliative care unit, $23 \%$ of patients receiving palliative sedation were discharged ${ }^{16}$.

\section{Monitoring}

Unfortunately, there are no specific scales to assist in the management of sedation depth in terminally ill patients. Thus, many clinicians have used scales, such as the Ramsay and Richmond, for assessing the level of sedation-agitation, although they have not been validated for palliative sedation ${ }^{22}$.

Palliative sedation is safe and effective for most patients with terminal cancer and refractory symptoms ${ }^{37}$. However, complications (respiratory and circulatory) occur in small numbers of patients. Thus, it is necessary to compare studies of different types of sedation to determine the more effective and safer protocol ${ }^{37}$. 


\section{Nutritional support and hydration}

During the course of the terminal disease, the need for nutrition and hydration is variable. While there are numerous studies in literature on nutrition and hydration in patients with cancer in palliative care, there are no specific guidelines for terminal-stage patient. There is evidence that artificial nutrition will prolong the life of these patients, and due to the associated risks, some authors do not suggest that artificial nutrition be prescribed for these patients, which should be banned from the moment sedation is started $24,39,40$. In clinical practice, enteral nutrition is often maintained.

The opinions on hydration in terminally ill patients are varied. In a systematic review of the literature on the subject, the author concluded that hydration is not beneficial or harmful to these patients. Some authors argue against the use of fluid in terminally ill patient because water restriction can result in decreased pulmonary, salivary, or gastrointestinal secretions and, thus, reduce coughing, vomiting, and the need for interventions such as aspiration for relief. Less fluid administration results in decreased urine output and, therefore, less need for bladder catheterization. Theoretically, there is also a decrease in symptoms caused by ascites and edema around the tumor. Proponents of hydration in terminally ill patients say that in well-hydrated patient there is lower risk of sedation, seizures, constipation, pressure sores, dry mouth, and opioid toxicity, especially if kidney failure occurs. Some authors also believe that hydration decreases the incidence of delirium, although two randomized studies found no influence of hydration on its occurrence. Some authors believe that in patients whose death is expected for more than a week, dehydration can hasten it 41 .

The legal, ethical and cultural implications of fluid and nutritional support in palliative care of patients should also be considered. Although fluid and nutritional support may be physiologically unnecessary in these circumstances, is maintenance may lead to cultural and psychological benefits. The heterogeneity of attitudes toward hydration in terminally ill patients reflects the lack of consensus in expert opinion. There is no evidence to support either view ${ }^{24}$.

In a palliative care unit, only 3 out of 266 patients received artificial hydration after the beginning of sedation ${ }^{9}$. In one study, $63 \%$ of patients received sedation after hydration, and the remainder did not receive it due to fluid retention or patient's request ${ }^{17}$.

Decision on whether or not to hydrate the patient should be taken separately from the sedation, and provide it if benefit is greater than damage ${ }^{8}$.

\section{Palliative sedation for patient care at home}

Many patients prefer to be at home at the time of death and, therefore, palliative sedation for outpatients may be an important area of research. However, few studies have been published on the subject.

Despite being much propagated, the idea that sedation at home is better because patient is comforted among family members, the author of a review article found that it is performed in $5-36 \%$ of patients ${ }^{13}$.

Some authors consider that patients with refractory and unbearable symptoms, with short life expectancy, who reside less than 20 minutes from the hospital, and are already being assisted by a medical team for a long period, preferably during all stages of disease, are candidates for palliative sedation at home. They also considered as criteria to indicate sedation at home the absence of any other sources of suffering for the family, such as serious chronic disease, alcoholism, and drug abuse ${ }^{42}$.

For palliative sedation at home, a protocol for induction and maintenance may be followed. Intravenous bolus of midazolam $0.07 \mathrm{mg} \cdot \mathrm{kg}^{-1}$ may be used to induce sedation. Maintenance is initiated with midazolam $1 \mathrm{mg} \cdot \mathrm{h}^{-1}$ administered subcutaneously; in case of failure, it is increased to $2 \mathrm{mg}^{-1} \mathrm{~h}^{-1}$. If it still fails, chlorpromazine $3 \mathrm{mg} \cdot \mathrm{h}^{-1}$ and promethazine $3 \mathrm{mg} \cdot \mathrm{h}^{-1}$ are added; in case of failure, midazolam $2 \mathrm{mg} \cdot \mathrm{h}^{-1}$, chlorpromazine $6 \mathrm{mg} \cdot \mathrm{h}^{-1}$, and promethazine $6 \mathrm{mg} \cdot \mathrm{h}^{-1}$ are administered. According to some authors, physician and nurse must follow the induction of sedation ${ }^{3,43}$.

Sedation level should be monitored twice daily during induction and after stabilization by physician or nurse using Ramsay's scale of sedation. Sedation failure is considered if score on Ramsay's scale is less than 5 after 12 hours of treatment ${ }^{22,42}$.

Opioids should be maintained and dose adjusted when pain is not controlled. The opioid of choice is morphine because dose titration is easier. Transdermal or oral opioids should be switched to morphine in equivalent doses ${ }^{38,42}$.

The patient must be hydrated, but in a restrictive manner, usually up to $1,000 \mathrm{~mL}$ of saline per day. Urinary catheter should be placed to control diuresis and prevent urinary retention ${ }^{41,42}$. During sedation, the medical and nursing staff must be on call for emergencies ${ }^{42}$.

\section{CONCLUSION}

Although palliative sedation has been lately considered a normal medical practice, there are still many gaps in our current 
understanding. There is no consensus about which are the standard drugs, maintenance or not of food, fluid intake, and hydration. Moreover, there is no ethical clarification on possible life-shortening effects and decision-making process. It is important to define explicit criteria and conditions for the use of sedation in terminally ill patient in order to contribute to good medical practice in this field.
For now, it is essential that there is debate on the subject, and that doctors and other health professionals are aware of the rules and starting points in order to provide the patient, facing the end of life, with appropriate treatment that may ease his pain and suffering. 
5. Kettler D, Nauck F - Palliative care and involvement of anaesthesiology: current discussions. Curr Opin Anaesthesiol, 2010;23:173-176.

6. Hoven B. What to do when a competent ICU patient does not want to live anymore but is dependent on life-sustaining treatment? Experience from the Netherlands. Intensive Care Med, 2010;36:2145-2148.

7. Morita $T$, Tsuneto $S$, Shima $Y$ - Definition of sedation for symptom relief: a systematic literature review and a proposal of operational criteria. J Pain Symptom Manage, 2002; 24(4):447-453.

8. De Graeff A, Dean M-Palliative sedation therapy in the last weeks of life: a literature review and recommendations for standards. J Palliat Med, 2007;10(1):67-85.

9. Claessens $P$, Menten $J$, Schotsmans $P$ et al. - Palliative sedation, not slow euthanasia: A prospective, longitudinal study of sedation in Flemish Palliative Care Units. J Pain Symptom Manage, 2010 Sep 9. [Epub ahead of print].

10. Vitetta L, Kenner D, Sali A - Sedation and analgesia-prescribing patterns in terminally ill patients at the end of life. Am J Hosp Pall Care, 2005;22:465-473.

11. Sykes N, Thorns A - Sedative use in the last week of life and the implications for end-of-life decision making. Arch Intern Med, 2003;163:341344.

12. Mercadante $S$, Intravaia G, Villari $P$ et al. - Controlled sedation for refractory symptoms in dying patients. J Pain Symptom Manage, 2009;37(5):771-779.

13. Mercadante S, Porzio G, Valle A et al. - Palliative Sedation in Patients with Advanced Cancer Followed at Home: A Systematic Review. J Pain Symptom Manage. 2011 Jan 11. [Epub ahead of print]

14. Alonso-Babarro A, Varela-Cerdeira M, Torres-Vigil I et al. - At-home palliative sedation for end-of-life cancer patients. Palliat Med, 2010;24(5):486-492.

15. Rosengarten OS, Lamed Y, Zisling T, Feigin A, Jacobs JM. - Palliative sedation at home. J Palliat Care, 2009;25(1):5-11.

16. Elsayem A, Curry lii E, Boohene $\mathrm{J}$ et al. - Use of palliative sedation for intractable symptoms in the palliative care unit of a comprehensive cancer center. Support Care Cancer, 2009;17(1):53-59.

17. Morita $\mathrm{T}$, Chinone $\mathrm{Y}$, Ikenaga $\mathrm{M}$ et al. - Ethical validity of palliative sedation therapy: a multicenter, prospective, observational study conducted on specialized palliative care units in Japan. J Pain Symptom Manage, 2005;30(4):308-319.

18. Kohara $\mathrm{H}$, Ueoka $\mathrm{H}$, Takeyama $\mathrm{H}$ et al. - Sedation for terminally ill patients with cancer with uncontrollable physical distress. J Palliat Med, 2005;8(1):20-25.

19. Santasuanan AV, Jiménez NC, Salvador $S$ et al. - The final week of life in an acute care hospital: review of 401 consecutive patients. Rev Esp Geriatr Gerontol, 2008;43(5):284-290.

20. Marín JLM, Reta IS, Campos RAI et al. - Treatment recommendations at the end of the life of the critical patient. Med Intens, 2008;32(3):121133.

21. Russell JA, Williams MA, Drogan O - Sedation for the imminently dying: survey results from the AAN Ethics Section. Neurology, 2010;74(16):1303-1309.

22. Rousseau $P$ - Palliative sedation in the management of refractory symptoms. J Support Oncol, 2004; 2:181-186.

23. Stiel S, Krumm N, Schroers $\mathrm{O}$ et al. - [Indications and use of benzodiazepines in a palliative care unit]. Schmerz, 2008;22(6):665-671.

24. De Graeff A, Dean M - Palliative sedation therapy in the last weeks of life: a literature review and recommendations for standards. J Pall Med, 2007;10:67-85.

\section{REFERENCES}

1. Muller-Busch $\mathrm{HC}$, Andres I, Jehser T - Sedation in palliative care- a critical analysis of 7 years experience. BMC Palliat Care, 2003;2(1):2.

2. Gonçalves JAF - Sedation and Expertise in Palliative Care. J Clin Onc, 2006;24(25):44-45.

3. Verkerk $M$, van Wijlick $E$, Legemaate $\mathrm{J}$ et al. - A national guideline for palliative sedation in the Netherlands. J Pain Symptom Manage, 2007;34(6):666-670.

4. Claessens P, Menten J, Schotsmans $P$ et al. - Palliative sedation: A review of the research literature. J Pain Symptom Manage, 2008;36(3):310-333.

25. Rosengarten OS, Lamed $\mathrm{Y}$, Zisling $\mathrm{T}$ et al. - Palliative sedation at home. J Palliat Care, 2009;25(1):5-11.

26. Krakauer EL, Penson RT, Truog RD et al. - Sedation for intractable distress of a dying patient: acute palliative care and the principle of double effect. Oncologist, 2000;5:1:53-62

27. Mercadante S, Ferrera $P$, Casuccio $A$ - The use of opioids in the last week of life in an acute palliative care unit. Am J Hosp Palliat Care. 2010;27(8):514-517.

28. Hasselaar JGJ, Verhagen SCAHHVM, Wolff AP et al. - Changed patterns in Dutch palliative sedation practices after the introduction of a National Guideline. Arch Intern Med, 2009;169(5):430-437. 
29. Von Roenn JH, Von Gunten CF - Are We Putting the Cart Before the Horse? Arch Intern, 2009;169(5):429.

30. Morita T, Tsunoda, J, Inoue S, Chihara S et al. - Effects of high dose opioids and sedatives on survival in terminally ill cancer patients. $J$ Pain Symptom Manage, 2001;21(4):281-289.

31. Berger JM, Ryan A, Vadivelu $\mathrm{N}$ et al. - Ketamine-fentanyl-midazolam infusion for the control of symptoms in terminal life care. Am J Hosp Palliat Care, 2000;17(2):127-132.

32. Okon $T$ - Ketamine: an introduction for the pain and palliative medicine physician. Pain Phys, 2007;10:493-500.

33. Carter MJ, Thomas S, Forbes $\mathrm{K}$ et al. - Ketamine: does it have a role in palliative sedation? J Pain Symptom Manage, 2008;36:1-3

34. Herndon CM, Zimmerman E - High-dose propofol drip for palliative sedation: a case report. Am J Hosp Palliat Care, 2008 Dec-2009;25(6):492-495.

35. McWilliams K, Keeley PW, Waterhouse ET - Propofol for terminal sedation in palliative care: a systematic review. J Palliat Med, 2010;13(1):73-76.

36. Sykes N, Thorns A - Sedative use in the last week of life and the implications for end-of-life decision making. Arch Intern Med, 2003;163(3):341-344.

37. Morita $\mathrm{T}$, Chinone $\mathrm{Y}$, Ikenaga $\mathrm{M}$ et al. - Efficacy and safety of palliative sedation therapy: a multicenter, prospective, observational study conducted on specialized palliative care units in Japan. J Pain Symptom Manage, 2005;30(4):320-328.

38. Américo $\mathrm{AFQ}$ - As últimas 48 horas de vida. Em: Carvalho RT Manual de Cuidados Paliativos. Rio de Janeiro, Editora Diagraphic, 2010;290-298.

39. Hawryluck LA, Harvey WR, Lemieux-Charles L, Singer PA. Consensus guidelines on analgesia and sedation in dying intensive care unit patients. BMC Med Ethics, 2002;3:1-9.

40. Quill TE, Lo B, Brock DW - Palliative options of last resort: a comparison of voluntarily stopping eating and drinking, terminal sedation, physician-assisted suicide, and voluntary active euthanasia. JAMA, 1997;278:2099-2104.
41. Craig GM - On withholding nutrition and hydration in the terminally ill: has palliative medicine gone too far? J Med Ethics, 1994;20:139-143.

42. Porzio G, Aielli F, Verna L et al. - Efficacy and safety of deep, continuous palliative sedation at home: a retrospective, single-institution study. Support Care Cancer, 2010:18:77-81. 